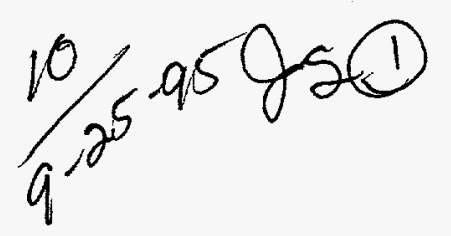

\title{
TRANSIENT BEAM LOADING IN THE SLC DAMPING RINGS*
}

\author{
M.G. Minty, R.H. Siemann \\ Stanford Linear Accelerator Center, Stanford University, Stanford, CA 94309 USA
}

\section{Abstract}

Effects arising from heavy beam loading in the Stanford Linear Collider (SLC) Damping Rings are described. The stability of the rf system and particle beam is studied using a numerical model of the beam cavity interaction with multiple feedback loops. Nonlinearities of the power source are also considered. The effects of beam-induced transients and intensity jitter on the rf system are analyzed and used to determine stability tolerances for both high current and low current pulses.

\section{INTRODUCTION}

When a particle beam is injected into or extracted from a circular accelerator, beam-induced electromagnetic fields cause the accelerating cavity voltage to oscillate at the synchrotron frequency. For fast cycling storage rings like the SLC damping rings, the magnitude of these oscillations depends on the time between extraction and injection of beam pulses, the cavity fill time, and on the beam current. At the SLC where the single bunch beam current was increased by $20 \%$ for the recent run, transient loading occassionally caused rf system and beam instabilities. To better understand the sources of these instabilities, simulations ${ }^{1,2}$ were performed using Matrix $_{x}{ }^{3}$, a program developed for the analysis of dynamic stability. The simulations included a detailed model ${ }^{2}$ of the rf system for which a block diagram is shown in Fig. 1.

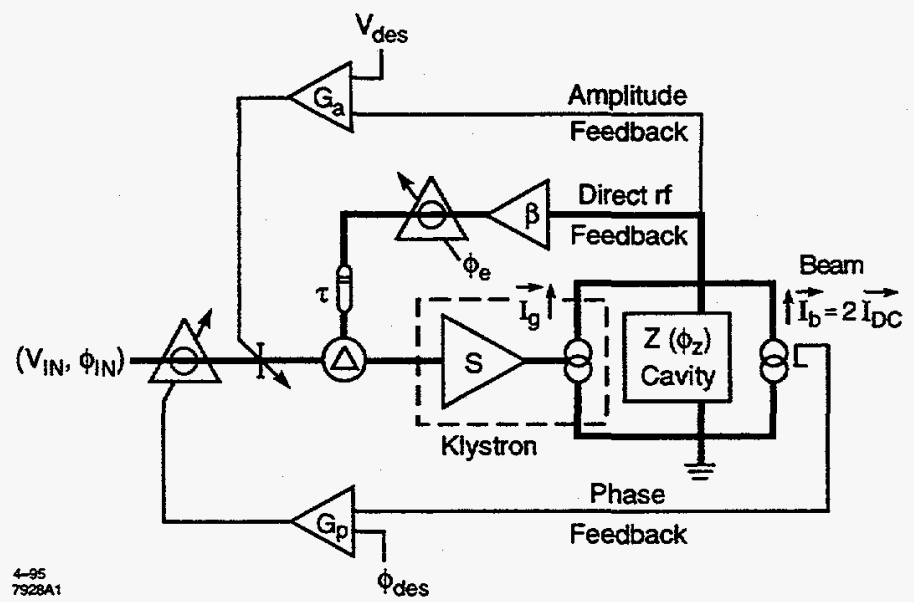

Figure. 1. Circuit model of a beam loaded rf system including amplitude, phase, and direct If feedback. The thick solid lines indicate paths for phasors which have both an amplitude and phase.

*Work

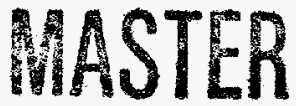

supported by Department of Energy contract DEAC0376SF00515.

\section{TRANSIENT BEAM LOADING}

Large oscillations in the cavity voltages induced by injection of a high current beam in the SLC damping rings resulted in substantial beam losses ${ }^{4}$, which were reduced using direct rf feedback ${ }^{5}$. Figure 2 shows simulation results with direct rf feedback (open loop gain $H=6$ ) and without. With feedback, the cavity fill time is reduced by $1+H$. The cavity therefore responds quickly to the voltage and phase errors resulting from the absence of beam. Residual oscillations still persist however because the klystron is saturated when the beam is injected.

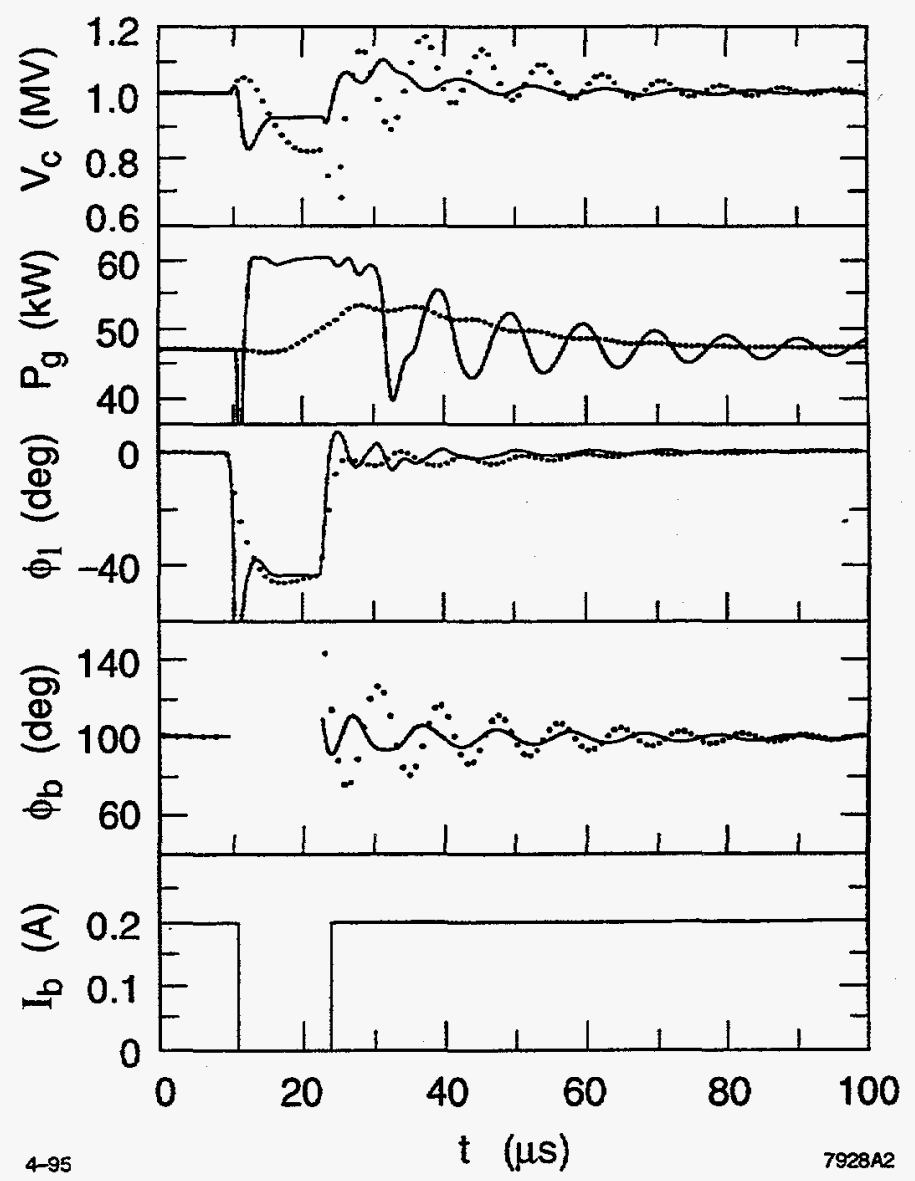

Figure. 2. Simulations of transient beam loading. The cavity voltage $V_{c}$, the klystron output power $P_{g}$, the loading angle $\phi_{l}$, the beam phase $\phi_{b}$, and current $I_{b}$ are plotted as a function of time for a direct rf feedback open loop gain of $H=0$ (dotted curves) and $H=6$ (solid curves).

A second problem during operation was poor regulation of the cavity voltage. Loss of regulation can result from beam-induced transients: if the klystron input power is not properly limited, there exist two solutions for the feed- 
back loops. One solution, on the rising edge of the klystron power curve is stable. The other, beyond saturation, is unstable. Simulation results are shown in Fig. 3 (compare with experimental data in Ref. 4). At each beam current, the tuning angle was adjusted to maintain a zero loading angle, $\phi_{l}=0$, in the steady state with beam. At high beam current more power is required in the steady state and the small signal gain approaches zero; just below saturation, any change in the klystron input power produces little effect on the output power. At high beam current the klystron is unable to return to a condition of positive small signal gain and the cavity voltage is not maintained as a result.

Intensity fluctuations of the incoming beam are considered tolerable provided the rf cavity voltage can regulate. For a fast cycling storage ring with a store time much smaller than the bandwidth of the tuner feedback loops, the effect of injecting a pulse of different current is a change in the steady state loading angle $\phi_{l}$. This in turn requires more klystron power. As discussed previously, the rf system is stable provided that the combined effects of the transients induced by extraction and injection of the beam are such that the klystron returns to a state of positive small signal gain.
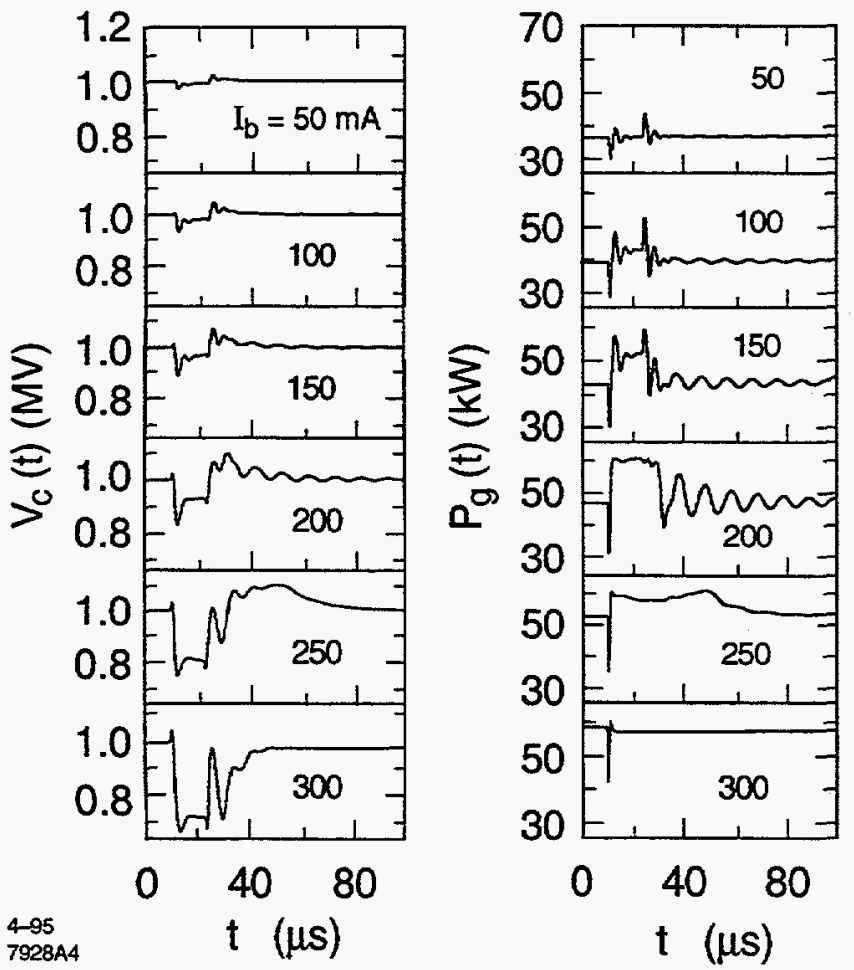

Figure. 3. Transient beam loading as a function of $I_{b}$ with $\phi_{l}=0$ in the steady state.

\section{STABILITY TOLERANCES}

The percentage tolerable current jitter $\left|\frac{\delta I_{b}}{I_{b}}\right|$ as a function of $I_{b}$ when $\phi_{l}=0$ is plotted in Fig. 4. In these simulations the steady state current $I_{b}$ is extracted, and a new pulse of current $\delta I_{b}+I_{b}$ is injected. The rf system is easily able to regulate for large fluctuations in the beam current except at the highest currents. With $I_{b}=0.20 \mathrm{~A}$ for example, the rf system would remain stable if a subsequent pulse of $40 \%$ above nominal, or $0.28 \mathrm{~A}$, were injected into the ring. The vertical line at $I_{b}=I_{m}$, however, indicates a hard limit due to a missing pulse. With $I_{b}>I_{m}$ the klystron is driven towards saturation when the beam is absent. A subsequent pulse may then experience an arbitrary cavity voltage. The time required for the system to become stable depends on the response time of the slow tuner feedback loops and on the output of low level amplifiers which may limit the maximum klystron input power. This hard limit presents a strict limit on the maximum current. At the SLC with a cavity voltage of $1 \mathrm{MV}$, a $60 \mathrm{~kW}$ maximum klystron output power, and the cavities tuned to $\phi_{l}=0$, stability against missing pulses is ensured for $I_{b}<0.16 \mathrm{~A}$. To accomodate $0.22 \mathrm{~A}$ rf currents for the 1994 run, the available operating region was expanded by lowering the rf gap voltage from $1 \mathrm{MV}$ to $800 \mathrm{kV}$.

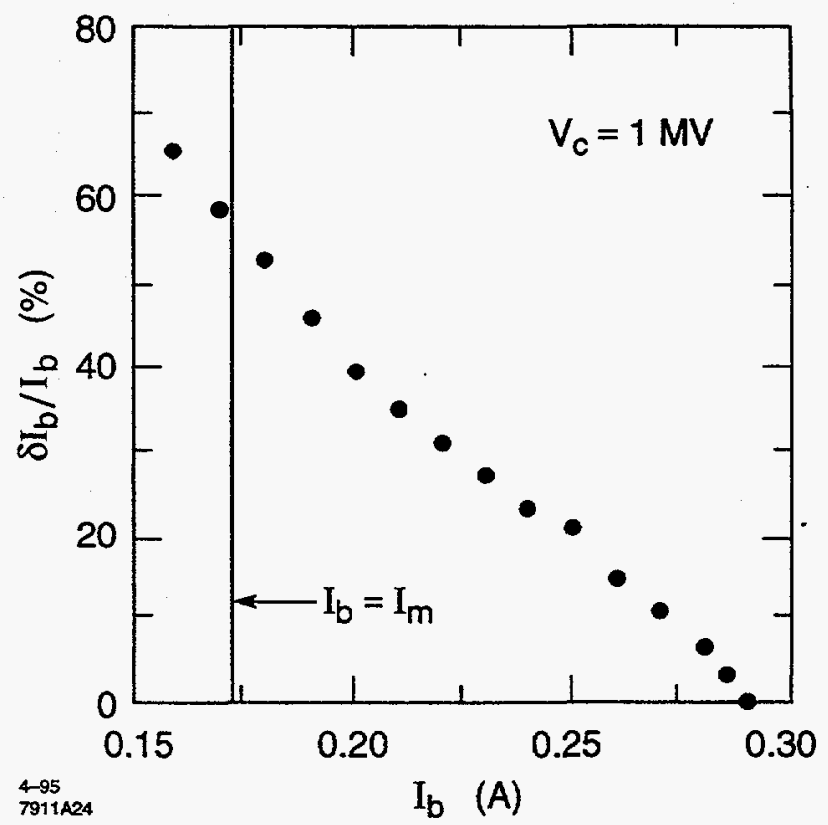

Figure. 4. Tolerable current jitter $\frac{\delta I_{b}}{I_{b}}$ as a function of nominal current $I_{b}$ with $\phi_{l}=0$ in the steady state.

The parameter space for voltage regulation in the steady state is shown in Fig. 5 for the rf operating conditions of the past 3 years. The solid curve is an analytic result ${ }^{6}$ for the contour of constant klystron power at the indicated power and cavity voltage. The small circles are simulation ${ }^{2}$ results using a nonlinear klystron and a power limiter. The large dots indicate the operating current and tuning angle. In general, the highest beam currents are obtainable for $\phi_{l}=0$. The limitation due to low current or missing pulses is representable by a vertical line intersecting the contour of maximum klystron output power and $I_{b}=0$. If not properly compensated, the if system can be unstable for operation to the left of this line. Alternatively, the performance requirements of the klystron can be specified using this analysis ${ }^{6}$. 


\section{DISCLAIMER}

This report was prepared as an account of work sponsored by an agency of the United States Government. Neither the United States Government nor any agency thereof, nor any of their employees, makes any warranty, express or implied, or assumes any legal liability or responsibility for the accuracy, completeness, or usefulness of any information, apparatus, product, or process disclosed, or represents that its use would not infringe privately owned rights. Reference herein to any specific commercial product, process, or service by trade name, trademark, manufacturer, or otherwise does not necessarily constitute or imply its endorsement, recommendation, or favoring by the United States Government or any agency thereof. The views and opinions of authors expressed herein do not necessarily state or reflect those of the United States Government or any agency thereof. 


\section{DISCLAIMER}

Portions of this document may be illegible in electronic image products. Images are produced from the best available original document. 


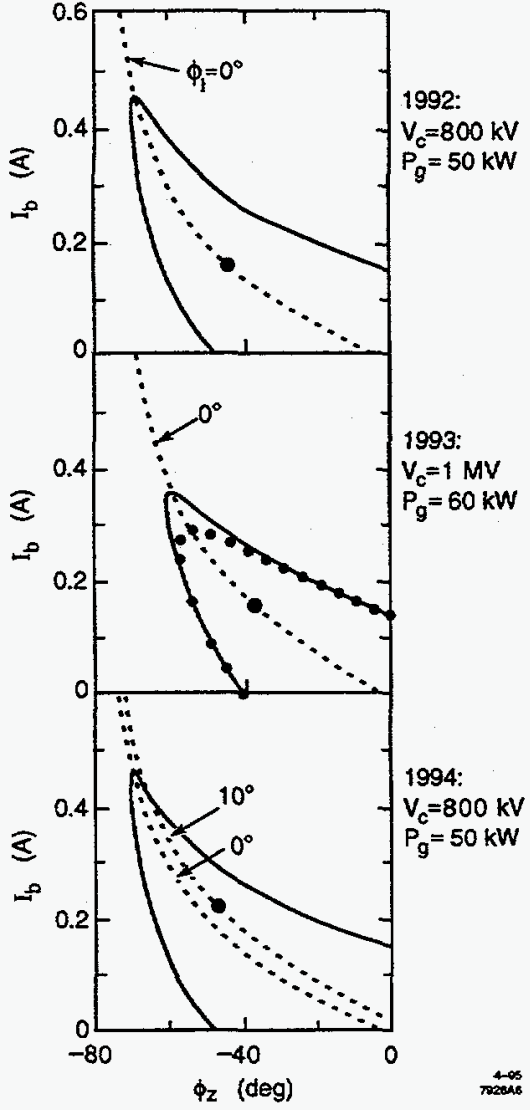

Figure. 5. Parameter space for voltage regulation for SLC damping ring parameters for the past 3 years.

\section{A. Cavity Detuning}

Provided that the additional power reflected from the cavities is absorbed in an isolator between the cavities and klystron, intentional detuning $\left(\phi_{l} \neq 0\right)$ is advantangeous for two reasons. First, it provides stability against beaminduced transients as shown in Fig. 6. Notice that the peak-to-peak amplitude of the voltage oscillations is largest for $\phi_{l}=0$. This is due to the large difference in the klystron output power in the steady state and in saturation. Second, cavity detuning may be used to relax the hard limit from low current or missing pulses as evidenced by the change (see Fig. 5) in operating point, from $\phi_{l}=0$ to $\phi_{l}=10^{\circ}$, for the 1994 run.

\section{B. RF Conditioning}

To achieve higher beam currents for the same maximum klystron output power and cavity voltage, the klystron must be made to operate more efficiently; the hard limit due to missing pulses or absence of beam must be overcome. This can be accomplished by changing the voltage and phase references to the direct rf feedback loop. The required changes are calculable ${ }^{6}$. In practice, with a power limited klystron, the amount by which to lower the voltage reference would be to that value for which the control voltage from the amplitude feedback loop is unchanged by the change in beam current at injection. The amount by which to change the phase reference would be to minimize the change in the cavity voltage phase angle.

\section{CONCLUSION}

Simulation results were used to explain performance and performance limits and to suggest changes that could be made. At the SLC, rf system and beam instabilities were shown to result from the interaction of of feedback loops and a power limited klystron. Rf conditioning was suggested for future operations at even higher beam currents.
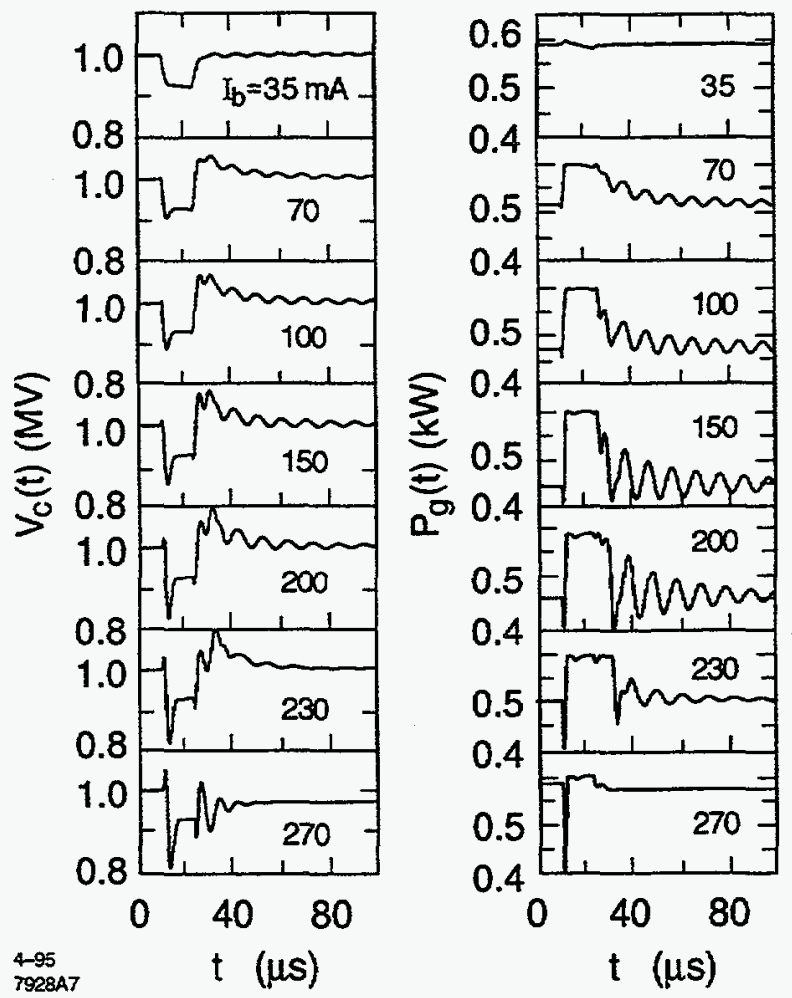

Figure. 6. Transient beam loading as a function of $I_{b}$ with $\phi_{z}=-45^{\circ}$ in the steady state.

\section{References}

[1] M.G. Minty et al, Proc. 1993 PAC 2373.

[2] M. G. Minty, R.H. Siemann, "Heavy Beam Loading in Storage Ring RF Systems', to be submitted to Nucl. Instr. and Methods.

[3] Matrix by Integrated System, Santa Clara, CA 95054 .

[4] M. G. Minty, et al., WAB12 1995 PAC.

[5] F. Pederson, IEEE Tran. on Nucl. Sci., NS-32, No. 3 (1985) 2138.

[6] M.G. Minty, R. H. Siemann, TPA09 1995 PAC. 